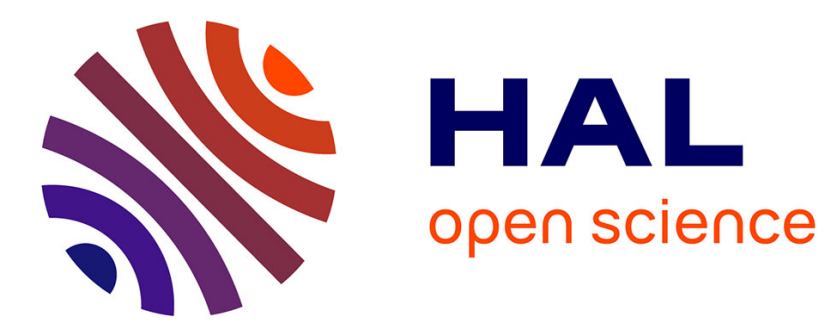

\title{
Labelling Sulcal Graphs Across Indiviuals Using Multigraph Matching
}

N. Buskulic, François-Xavier Dupé, S. Takerkart, Guillaume Auzias

\section{To cite this version:}

N. Buskulic, François-Xavier Dupé, S. Takerkart, Guillaume Auzias. Labelling Sulcal Graphs Across Indiviuals Using Multigraph Matching. 2021 IEEE 18th International Symposium on Biomedical Imaging (ISBI), Apr 2021, Nice, France. pp.1486-1490, 10.1109/ISBI48211.2021.9434035 . hal03401832

\section{HAL Id: hal-03401832 \\ https://hal.science/hal-03401832}

Submitted on 25 Oct 2021

HAL is a multi-disciplinary open access archive for the deposit and dissemination of scientific research documents, whether they are published or not. The documents may come from teaching and research institutions in France or abroad, or from public or private research centers.
L'archive ouverte pluridisciplinaire HAL, est destinée au dépôt et à la diffusion de documents scientifiques de niveau recherche, publiés ou non, émanant des établissements d'enseignement et de recherche français ou étrangers, des laboratoires publics ou privés. 


\title{
LABELLING SULCAL GRAPHS ACROSS INDIVIUALS USING MULTIGRAPH MATCHING
}

\author{
N. Buskulic ${ }^{\star}$, F.X. Dupé $\dagger$, S. Takerkart ${ }^{\star}$, G. Auzias ${ }^{\star}$ \\ *Institut de Neurosciences de la Timone UMR 7289, Aix-Marseille Université, CNRS \\ ${ }^{\ddagger}$ Aix Marseille Université, Unité de recherche, Institut Marseille Imaging \\ ${ }^{\dagger}$ Laboratoire d'Informatique et Systèmes UMR 7020, Aix-Marseille Université, CNRS
}

\begin{abstract}
The problem of inter-individual comparison is of major importance in neuroimaging to detect patterns indicative of neurological pathology. Few works have been addressing the comparison of individual sulcal graphs in which variations across subjects manifest as changes in the number of nodes, graph topology and in the attributes that can be attached to nodes and edges. Here, we quantitatively evaluated different graph matching approaches in both the pairwise and multigraph matching frameworks, on synthetic graphs simulating the structure and attributes distributions of real data. Our results show that multigraph matching approach outperforms pairwise techniques in all simulations. The application to a set of real sulcal graphs from 134 subjects confirms this observation and demonstrates that multigraph matching approaches can scale and have a great potential in this context.
\end{abstract}

\section{MRI}

Index Terms - Graph Matching, Brain, Morphometry,

\section{INTRODUCTION}

One area of great interest in neuroscience is the study of the geometry and pattern of the brain cortical folding (morphometry). Finding the best set of descriptors for the morphology of the brain is of utmost importance since the characterisation of putative biomarkers indicative of a neurological pathology is rooted on the definition of biologically relevant representation. Decomposing the cortical surface in sulcal basins and sulcal pits as proposed in [1, 2, 3] give access to promising morphological descriptors that can be naturally represented as sulcal graphs. As illustrated on Fig.1.A and described in details in [1], sulcal basins are defined as concavities in the white matter surface which are bounded by convex ridges, and the deepest point in each bassin defines the corresponding sulcal pit. A graph representation can then be constructed by considering each sulcal basin (or corresponding pit) as a node, while the edges connect each bassin to its adjacent neighoring basins which meet at the top of a ridge. See [2] for extensive description of sulcal graphs construction. Various geometrical information of a sulcal basin can then be attributed to graph nodes (such as e.g. the depth of the pit, its 3D position...), while the spatial organization of the basins is integrated into the edges and topology of the graph. The edges can also carry specific attributes such as the geodesic distance between two sulcal pits. Formally, the geometry of the cortical surface of any individual can be represented as a sulcal graph $\mathcal{G}$ as a triplet of vertex, edges and attributes, namely $\mathcal{G}=(V, E, A)$ with the cardinality of the graph being the number of basins also noted $|\mathcal{G}|=n$. In this work, we consider as attributes the 3D coordinates of the sulcal pits on the sphere for the nodes and the geodesic distance for the edges. As illustrated on Fig.1.B with the respective sulcal graphs from three subjects, variations across individuals manifest in the graphs as changes in the number of nodes, graph topology and in the attributes attached to nodes and edges.

Quantitatively comparing a set of brains is necessary to discover markers of diseases. One solution is to use an atlas as a common space between brains to compare them as e.g. in $[4,5]$. However, the process of building a good atlas and appropriate projection operator remains an open research question. More specifically, the "atlas-mapping" approach might be an over simplification of the problem since potentially relevant geometrical information is not taken into account, and in particular topological variations across individuals are ignored.

On the other side, comparing brains using sulcal graph matching appears relevant because all the geometrical information is integrated. Matching such graphs is however challenging due to the complexity of brain geometry that is preserved in graph representations. The specific challenges to be adressed in this context are: 1) the presence of noise due to imperfect sulcal basins segmentation, resulting from inacurate extraction of the cortical mesh; 2) no ground truth data exist at the scale of sulcal basins. Defining ground truth data is already a tedious and ambiguous task at the scale of sulci [6]. No anatomical nomenclature or validated atlas is available for sulcal basins, even the appropriate number of sulcal labels is unknown so far.

All this makes the problem of matching a pair of sulcal graphs an ill-posed problem. Indeed, the conjonction of major variations across individuals and non-negligible level of noise constitute clearly unfavorable settings for the pairwise graph matching techniques to perform well. However, multi- 
graph matching approaches might be efficient in this context. In principle, tackling the sulcal graph mathing problem at the group level might allow the comonalities across individuals to emerge while distinguishing the anatomically-relevant information from the noise. By exploiting the information from several brains together, the geometrical information that is shared by the majority of individuals will help to regularize the matching problem and allow to identify putative noisy graph nodes in a much more robust way than in the case of a pairwise matching between two subjects. As such, this type of techniques have the potential to 1) yield improved correspondences across brains and 2) define and unbiased, data-driven nomenclature of sulcal basins.

In this work, we evaluate a set of recently published pairwise and multigraph matching tehcniques $[7,8,9,10,11]$, in the context of sulcal graphs. In order to quantitatively compare the selected methods, we propose a procedure to generate a set of synthetic graphs simulating sulcal graphs representative of a population. We then test the assumption that multigraph matching approaches are more robust than pairwise techniques to specifically desgined types of noise. An application to real-world data from 134 subjects coroborates our observations on simulations and demonstrates the scalability of multigraph matching techniques.

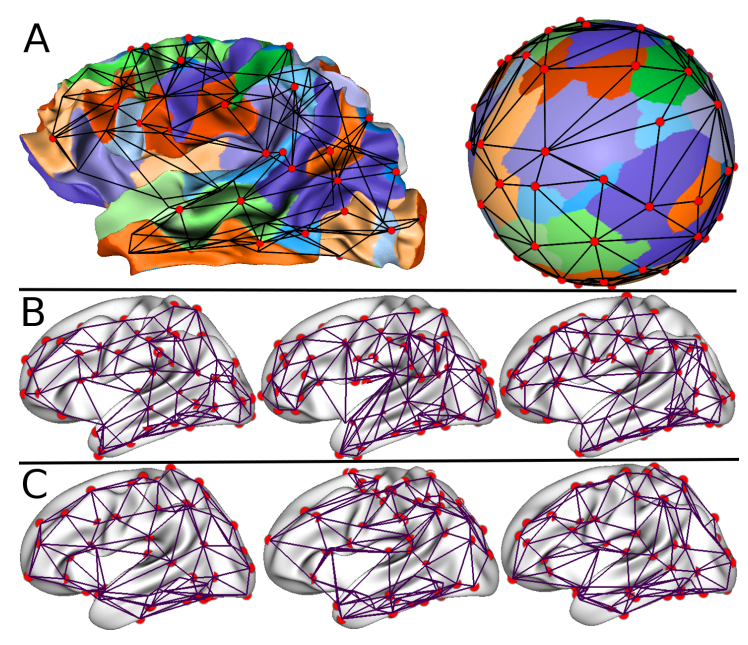

Fig. 1. A: An illustration of the sulcal graph from an individual on the cortical surface (left) and on the spherical domain (right). B: Sulcal graphs from three individuals mapped onto an average surface to show the variations in nodes number, attributes and graph topology. C: Simulated sulcal graphs generated using the procedure introduced in section 3.1.1.

\section{METHODS}

\subsection{Pairwise graph matching}

Graph matching refers to the problem of finding correspondences between the vertices of two graphs, ie estimating the assignment matrix $\mathbf{X}_{12} \in\{0,1\}^{n_{1} \times n_{2}}$ between the nodes of $\mathcal{G}^{1}$ and $\mathcal{G}^{2}$, with $n_{1}$ and $n_{2}$ being the number of nodes in each graph respectively. The largest part of the wide literature that deals with this problem solves the graph matching problem by maximising the affinity between the two graphs, i.e the summation of all vertex-to-vertex and edge-to-edge similarity values. We define the affinity matrix $\mathbf{K} \in \mathbb{R}^{n_{1} n_{2} \times n_{1} n_{2}}$ that encodes the affinity between graphs $\mathcal{G}^{1}$ and $\mathcal{G}^{2}$ : its diagonal represents the node-to-node similarities, while the off-diagonal terms contain edge-toedge affinity. In this work, the affinity matrix is based on a Gaussian kernel applied to the squared norm of two vectors of attributes as described in [2]. The pairwise graph matching can then be formalized as a quadratic assignment problem [12] $\max _{\mathbf{x}} \mathbf{x}^{\top} \mathbf{K} \mathbf{x}$, where $\mathbf{x}$ is the column-wise vectorized version of $\mathbf{X}_{12}$, with the following constraints: $\mathbf{X}_{12} \mathbf{1}_{n_{2}}=\mathbf{1}_{n_{1}}, \mathbf{X}_{12}^{\top} \mathbf{1}_{n_{1}} \leq \mathbf{1}_{n_{2}}, \mathbf{X}_{12} \in\{0,1\}^{n_{1} \times n_{2}}$. This formulation allows the two graphs to have different number of nodes $(n 1 \neq n 2)$. In this work, we apply a widely adopted technique that consists in adding dummy nodes to the smaller graph (i.e., adding slack variables to the assignment matrix and augment the affinity matrix by zeros). This processing enables the application of existing graph matching techniques which assume that all graphs are of equal sizes, and simplifies the formulation of multigraph matching techniques hereafter.

In practice, we selected several graph matching algorithms, representative of different classes of methods that have been proposed to solve this optimization problem. First, in SMAC [7], the discrete constraint is relaxed so that $\mathbf{X} \in \mathbb{R}^{n_{1} \times n_{2}}$, and solved using a spectral method by requiring that $\|\mathbf{x}\|_{2}=1$; a final discretization allows coming back into $\{0,1\}$. Secondly, in IPFP [8], an interior point method is used to solve the problem, which ensures that at each step of the algorithm, the solution is within the discrete space - which advantageously replace this final discretization. Thirdly, in RRWM [9], a Markov random walk is used on the association graph to match the constraints of the problem, which helps handling noisy graphs with outlier nodes. Finally, the recent KerGM approach [10] is based on a kernelized algorithm that is both memory- and computationnally-efficient, and yields state-of-the-art performances.

\subsection{Multi-Graph Matching}

When matching multiple graphs $\left\{\mathcal{G}^{1}, \cdots, \mathcal{G}^{N}\right\}$, such as in our problem of matching the sulcal graph of all individuals in a population, the standard solution consists in relying on a pairwise matching algorithm and applying it on all pairs of graphs. However, this solution can yield inconsistencies across multiple graphs, e.g combining the matchings of $\mathcal{G}^{1}$ to $\mathcal{G}^{2}$ and of $\mathcal{G}^{2}$ to $\mathcal{G}^{3}$ might not be equivalent to the direct matching of $\mathcal{G}^{1}$ to $\mathcal{G}^{3}$, especially in the presence of noise. In the literature, a handful of multi-graph matching methods attempt to overcome this problem by jointly finding the match- 
ings between all available graphs, i.e estimating the set of assignment matrices $\left\{\mathbf{X}_{i j}\right\}_{1 \leq i, j \leq N}$ that can be gathered in a bulk matrix:

$$
\mathbf{X}=\left(\begin{array}{cccc}
\mathbf{X}_{11} & \mathbf{X}_{12} & \cdots & \mathbf{X}_{1 N} \\
\vdots & \vdots & \ddots & \vdots \\
\mathbf{X}_{N 1} & \mathbf{X}_{N 2} & \cdots & \mathbf{X}_{N N}
\end{array}\right)
$$

As demonstrated in [13], the pairwise inconsistency can be minimized by ensuring the semi-definitiveness and the low-rankness of this bulk matrix. In the present study, we use the multi-graph matching algorithm described in [11], mALS, which exploits this property while allowing partial matching of nodes across graphs. It relies on a universe of nodes that each node in the bulk matrix can be matched to. This is particularly interesting when working with noisy graphs such as the sulcal graphs, for which the inter-individual differences that exist in cortical anatomy can yield different number of nodes across graphs. In order to optimize the matchings to the universe, mALS uses the ADMM method to minimize the following objective function :

$$
\min _{\mathbf{X}} \underbrace{-\sum_{i j}<\mathbf{K}_{i j}, \mathbf{X}_{i j}>}_{\text {node affinity }}+\underbrace{\alpha<\mathbf{1}, \mathbf{X}>}_{\text {sparsity }}+\underbrace{\lambda\|\mathbf{X}\|_{*}}_{\text {rank }}
$$

where $\mathbf{K}$ is the bulk matrix of nodes affinity, $<.$, . $>$ denotes the inner product and $\|\cdot\|_{*}$ is the nuclear norm. The first term favors matchings for which the nodes have large affinities, the second term of sparsity pushes toward a feasible solution and the nuclear norm term is a proxy function for the rank of the matrix, which helps minimizing the pairwise inconsistencies.

\section{EXPERIMENTS AND RESULTS}

\subsection{Simulations}

Our aim is to benchmark the techniques described above in the specific context of sulcal graphs. The major limitation here is the absence of ground truth data. Indeed, no nomenclature exists at the scale of sulcal basins, for which the anatomical ambiguity is problematic for a human expert. We overcome this obstacle by generating simulated graphs with corresponding ground truth matching, as a proxy of real-world graphs. We adapt the experimental protocol proposed in [14, $12,9]$ to create synthetic graphs that respect the following constraints regarding the structure, topology and attributes of our real data.

\subsubsection{Biologically plausible simulated graphs}

We observed from our real dataset (described in section 3.2) that the number of nodes for a sulcal graph is $88 \pm 5.0$. The first step of the generation procedure consits in creating a reference graph by randomly sampling 85 points on a sphere of radius 100 which is the domain where real-world graphs are projected before comparison across individuals (illustrated on Fig.1.A). The edges of the real graph represent the neighbourhood relationship between sulcal basins, assignating edges randomly across pairs of nodes would thus not be relevant. We propose to define the edges by computing the $3 \mathrm{D}$ convex hull of the previously sampled points on the sphere, forming a mesh connecting the nodes of the graph that respects the spatial adjacency on the spherical domain. The convex hull was computed using the QHull library (http://www.qhull.org) as available in scipy (https://scipy.org).

We then generate "noisy" graphs by perturbating the reference graph as follows: First, we add a Gaussian noise $z \sim$ $\mathcal{N}\left(0, \sigma^{2}\right)$ to the 3D coordinates of the nodes and reproject them onto the sphere. Second, we add $o$ outlier nodes sampling additional random points on the spherical domain. Then each outlier node is connected to a random number of its 10 nearest neighbours (which can be outliers as well). By setting the number of nearest neighbours to 10 , the probability of being connected to it is $\frac{\text { mean graph degree without outliers }}{10}$ which does not perturb too much the mean degree of the synthetic graph. Finally, we set the nodes and edges attributes as for real graph, i.e. the 3D coordinates for nodes and the geodesic distance between nodes for edges.

By modifying the parameters $\sigma$ and $o$, we can adapt the amount of noise in the nodes and edges attributes, and the in the graph topology respectively. As illustrated on Fig.1.C with $\sigma=50$ and $o=10$, this procedure generates simulated sulcal graphs that are highly consistent with real data and thus biologically plausible.

\subsubsection{Benchmark on simulated graphs}

We evaluated the algorithms with different sets of parameters of noise variance $\sigma$ and numbers of outliers $o$. For each set of simulation parameters, we generated 10 independent runs across which the metrics were averaged. In each run, we generated a family of 25 graphs with 85 inlier nodes with a noise variance of $\sigma$ and $o$ outliers nodes. We evaluate the performances of the different algorithms using the classical $F_{1}$-score:

$$
F_{1}=2 *(\text { precision } * \text { recall }) /(\text { precision }+ \text { recall })
$$

where precision and recall are defined as:

recall $=\frac{\text { number of correct matches found by the algorithm }}{\text { total number of correct matches between inliers }}$

precision $=\frac{\text { number of correct matches found by the algorithm }}{\text { total number of matches found by the algorithm }}$

This metric is more relevant when outlier nodes are present than the accuracy measure often used in the graph matching litterature. The highest possible value of $F_{1}$ is 1 , indicating perfect precision and recall, and the lowest possible value is 0 , if either the precision or the recall is zero. Fig. 2 shows the mean and standard deviation of the $F_{1}$-score across the 10 runs for each algorithm for the simulation with the number of outliers set to $o=20$ and the variance $\sigma$ of 
the noise on coordinates varying between 0 and 150 . We ran the same evaluation for a number of outliers $o$ equal to 0 and 10. The results were highly consistent across simulations parameters and the ordering of the different methods following their performance was always the same as in Fig.2.

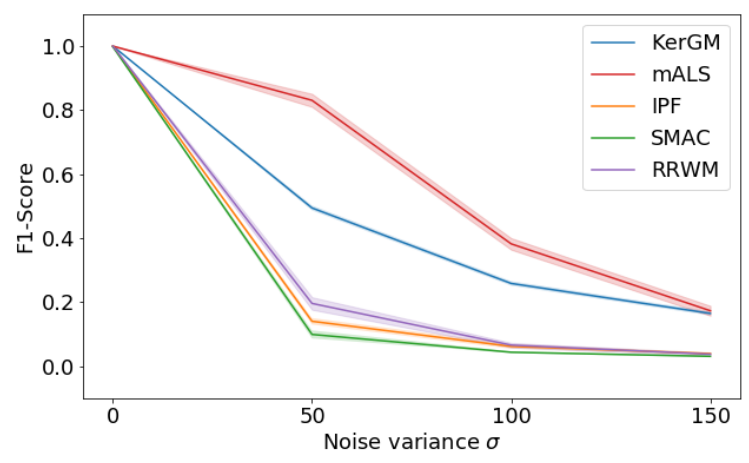

Fig. 2. Mean and standard deviation of $F_{1}$-score across 10 runs of 25 graphs with simulation parameters $o=20$ and $\sigma=0,50,100,150$.

This simulation-based evaluation shows that KerGM clearly outperforms the other pairwise graph matching techniques, with limited degradation of its performance when the level of noise increases compared to other pairwise approaches. RRWM is the second-best pairwise algorithm, with much lower performances than KerGM. The other pairwise algorithms IPF and SMAC provide poor results due to the presence of noise and outliers which they are not designed to deal with in such quantities.

Based on its performances and robustness to noise, we selected KerGM as the pairwise matching algorithm on top of which the multigraph matching approach mALS was applied. In addition, KerGM does not require the computation of a full affinity matrix which is crucial for the scalability in our context since we aim at matching simultaneously the sulcal graphs from hundreds of subjects.

The multigraph matching approach mALS outperformed all the pairwise techniques in every simulation settings, whatever the level of noise in nodes coordinates and number of outliers. When the noise becomes too large $(\sigma=150)$, the performances of $\mathbf{m A L S}$ degrades, but this extreme level of noise is not plausible for real data. These observations clearly support the assumption that tackling the problem of sulcal graph matching at the population level is more efficient than the pairwise approach.

\subsection{Real data}

For the evaluation on real data, we selected a set of 134 young adults healthy subjects, all right-handed, from the pubilcly available OASIS database [15]. See [1,2] for more details regarding brain tissues segmentation, mesh extraction and sulcal graphs construction. The metrics used on simulated graphs cannot be applied in this context since no ground truth matching is available. Following [16], we computed the node consistency defined as:

Given the set $\{\mathcal{G}\}_{1}^{N}$ and the bulk matrix $\mathbf{X}$, for nodes $\left\{\mathcal{N}_{u^{k}}\right\}_{u^{k}=1}^{n}$ in graph $\mathcal{G}_{k}$, its consistency is defined by

$C\left(u^{k}, X\right)=1-\frac{\sum_{i=1}^{N-1} \sum_{j=i+1}^{N}\left\|\mathbf{Y}\left(u^{k},:\right)\right\|_{F} / 2}{N(N-1) / 2}, \in(0,1]$,

where $\|\cdot\|_{F}$ is the Frobenius norm, $\mathbf{Y}=\mathbf{X}_{k j}-\mathbf{X}_{k i} \mathbf{X}_{i j}$ and $\mathbf{Y}\left(u^{k},:\right)$ is the $u^{k}$ th row of matrix $\mathbf{Y}$.

This metric was computed for each node of each graph. On average across all nodes and graphs, the node consistency increased from 0.29 obtained with the best pairwise technique KerGM to 0.91 for the multigraph matching approach mALS. This consistutes a major improvement and confirms that the level of noise in real data is probably inferior to the case where $\sigma=100$ in the simulations, i.e. when mALS is much more efficient than pairwise techniques. The Fig.3 shows the node consistency averaged across graphs for each node, allowing to observe the spatial variations of the consistency across cortical regions. Most consistenly matched nodes are located in regions such as the central and postcentral sucli, intra-parietal sulcus and superior temporal region, which are known to be less variable across individuals than e.g. the frontal and anterior temporal regions. This pattern is consistent with the literature of inter-individual variability and supports the anatomical relevance of the correspondences across individual graphs resulting from the multigraph matching technique.

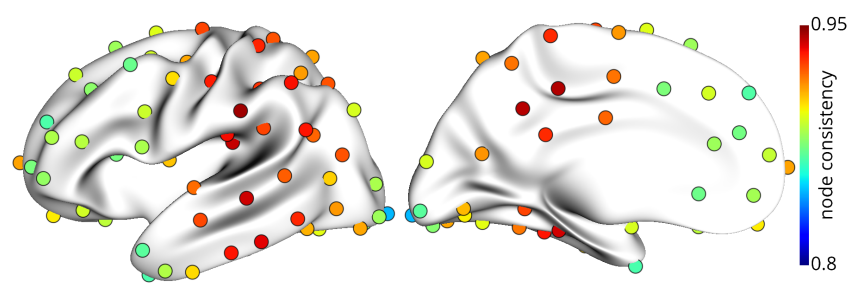

Fig. 3. Node consistency computed for each node of each graph with respect to the remaining graphs, and then averaged across graphs.

\section{CONCLUSION}

In this work, we set up a reallistic simulation framework allowing to quantitatively benchmark graph matching techniques for the challenging task of matching sulcal graphs across indivduals. Our results on both simulations and real data confirmed the superiority of multigraph matching approaches compared to pairwise techniques in this context. The application to real dataset of 134 subjects demonstrated the scalability and relevance of multigraph matching approaches. 


\section{ACKNOWLEDGMENTS}

The project leading to this publication has received funding from Excellence Initiative of Aix-Marseille University - A*MIDEX, a french "Investissements d'Avenir" program. Data used in the preparation of this article were obtained in part by the OASIS cross-Sectional with principal investigators D. Marcus, R, Buckner, J, Csernansky J. Morris; P50 AG05681, P01 AG03991, R01 AG021910, P50 MH071616, U24 RR021382, R01 MH56584. No funding was received for conducting this study. The authors have no relevant financial or non-financial interests to disclose.

\section{COMPLIANCE WITH ETHICAL STANDARDS}

This research study was conducted retrospectively using human subject data made available in open access by http://oasisbrains.org/. Ethical approval was not required as confirmed by the license attached with the open access data.

\section{REFERENCES}

[1] G. Auzias, L. Brun, C. Deruelle, and O. Coulon, "Deep sulcal landmarks: Algorithmic and conceptual improvements in the definition and extraction of sulcal pits," NeuroImage, vol. 111, pp. 12-25, May 2015.

[2] S. Takerkart, G. Auzias, L. Brun, and O. Coulon, "Structural graph-based morphometry: A multiscale searchlight framework based on sulcal pits," Medical Image Analysis, vol. 35, pp. 32-45, Jan. 2017.

[3] K. Im, R. Pienaar, J.-M. Lee, J.-K. Seong, Y.Y. Choi, K.H. Lee, and P.E. Grant, "Quantitative comparison and analysis of sulcal patterns using sulcal graph matching: a twin study.," NeuroImage, vol. 57, no. 3, pp. 1077-86, aug 2011.

[4] B. Fischl, Martin I Sereno, R B Tootell, and A. M. Dale, "High-resolution intersubject averaging and a coordinate system for the cortical surface.," Human brain mapping, vol. 8, no. 4, pp. 272-84, jan 1999.

[5] I. Kaltenmark, C. Deruelle, L. Brun, J. Lefèvre, O. Coulon, and G. Auzias, "Cortical inter-subject correspondences with optimal group-wise parcellation and sulcal pits labeling," Medical Image Analysis, 2020.

[6] L. Borne, D. Rivière, M. Mancip, and J.-F. Mangin, "Automatic labeling of cortical sulci using patch- or CNN-based segmentation techniques combined with bottom-up geometric constraints," Medical Image Analysis, vol. 62, pp. 101651, may 2020.

[7] T. Cour, P. Srinivasan, and J. Shi, "Balanced Graph Matching," in Advances in Neural Information Processing Systems 19, pp. 313-320. MIT Press, 2007.
[8] M. Leordeanu, M. Hebert, and R. Sukthankar, "An Integer Projected Fixed Point Method for Graph Matching and MAP Inference," in Advances in Neural Information Processing Systems 22, pp. 1114-1122. Curran Associates, Inc., 2009.

[9] D. Hutchison, T. Kanade, J. Kittler, J.M. Kleinberg, F. Mattern, J.C. Mitchell, M. Naor, O. Nierstrasz, Pandu R.C., B. Steffen, M. Sudan, D. Terzopoulos, D. Tygar, M.Y. Vardi, G. Weikum, M. Cho, J. Lee, and K.M. Lee, "Reweighted Random Walks for Graph Matching," in Computer Vision - ECCV 2010, vol. 6315, pp. 492-505. Springer Berlin Heidelberg, Berlin, Heidelberg, 2010, Lecture Notes in Computer Science.

[10] Z. Zhang, Y. Xiang, L. Wu, B. Xue, and A. Nehorai, "KerGM: Kernelized Graph Matching," in Advances in Neural Information Processing Systems 32, pp. 33353346. Curran Associates, Inc., 2019.

[11] X. Zhou, M. Zhu, and K. Daniilidis, "Multi-image Matching via Fast Alternating Minimization," in 2015 IEEE International Conference on Computer Vision (ICCV), Santiago, Chile, Dec. 2015, pp. 4032-4040, IEEE.

[12] S. Gold and A. Rangarajan, "A graduated assignment algorithm for graph matching," IEEE Transactions on Pattern Analysis and Machine Intelligence, vol. 18, no. 4, pp. 377-388, Apr. 1996.

[13] D. Pachauri, R. Kondor, and V. Singh, "Solving the multi-way matching problem by permutation synchronization," in Advances in Neural Information Processing Systems 26, C. J. C. Burges, L. Bottou, M. Welling, Z. Ghahramani, and K. Q. Weinberger, Eds., pp. 18601868. Curran Associates, Inc., 2013.

[14] F. Zhou and F. De la Torre, "Factorized graph matching," in 2012 IEEE Conference on Computer Vision and Pattern Recognition, Providence, RI, June 2012, pp. 127-134, IEEE.

[15] D.S. Marcus, T.H. Wang, J. Parker, J.G. Csernansky, J.C. Morris, and R.L. Buckner, "Open access series of imaging studies (oasis): Cross-sectional mri data in young, middle aged, nondemented, and demented older adults," Journal of Cognitive Neuroscience, vol. 19, no. 9, pp. 1498-1507, 2007.

[16] J. Yan, M. Cho, H. Zha, X. Yang, and S.M. Chu, "MultiGraph Matching via Affinity Optimization with Graduated Consistency Regularization," IEEE Transactions on Pattern Analysis and Machine Intelligence, vol. 38, no. 6, pp. 1228-1242, June 2016. 